\title{
HIV infection, and overweight and hypertension: a cross-sectional study of HIV-infected adults in Western Kenya
}

\author{
Akiko Saito ${ }^{1}$, Mohamed Karama ${ }^{2}$ and Yasuhiko Kamiya ${ }^{1 *}$ [D
}

\begin{abstract}
Background: Non-communicable diseases (NCDs) are increasing in Kenya, where HIV/AIDS remains a leading cause of death; however, few studies have investigated obesity and hypertension among adults with HIV infection. We conducted a cross-sectional study in Homa Bay, Western Kenya, during 2015 to determine the prevalence of overweight/obesity and hypertension among HIV-infected adults and to identify their risk factors.

Results: Anthropometric measurements and a structured questionnaire were administered to adults with HIV infection receiving care at Mbita Sub-county Hospital. A total of $251 \mathrm{HIV}$-positive individuals were enrolled. More women were overweight (17.2\%) and obese (3.6\%) than underweight (8.3\%). The prevalence of abdominal obesity was high in women (62.7\%), especially those aged 30-39 years. The prevalence of hypertension was $9.8 \%$ and $11.8 \%$ in men and women, respectively. Male participants tended to develop hypertension at an early age. Multivariate analysis showed that female sex was significantly associated with abdominal obesity. Regarding clinical factors, we identified an association between overweight and a history of opportunistic infections, as well as between hypertension and World Health Organization clinical stage. Sixty percent of HIV-infected participants assumed that a very thin body size indicated HIV infection.
\end{abstract}

Conclusions: The main findings of this study include a greater prevalence of overweight than underweight as well as a high prevalence of abdominal obesity among women. Social perception toward body size among people with HIV infection might remain problematic. Individuals living with HIV in Kenya should receive preventive intervention for overweight and abdominal obesity, with consideration of relevant social and cultural aspects.

Keywords: HIV, AIDS, Overweight, Obesity, Abdominal obesity, Hypertension, Body image, Kenya

\section{Introduction}

Non-communicable diseases (NCDs) have become an issue of worldwide concern and have been clearly identified as a problem to be addressed according to the Sustainable Development Goals by the United Nations [1]. Additionally, NCDs are predicted to cause half of all deaths in most developing countries by 2030 [2]. These countries are also disproportionately affected by communicable diseases [3]; therefore, preventive measures

\footnotetext{
* Correspondence: ykami@nagasaki-u.ac.jp

${ }^{1}$ School of Tropical Medicine \& Global Health, Nagasaki University, 1-12-4, Sakamoto, Nagasaki 852-8523, Japan

Full list of author information is available at the end of the article
}

are needed to minimize the double burden of communicable and non-communicable diseases.

Since anti-retroviral therapy (ART) was introduced to treat human immunodeficiency virus (HIV) infection, comorbidities affecting individuals with HIV infection have changed dramatically, with increasing prevalence of overweight and obesity [4] and NCDs [5]. HIV-infected individuals are no longer seriously affected by wasting syndrome; instead, the prevalence of overweight/obesity has been increasing $[4,6]$. Such changes necessitate measures to prevent NCDs that target individuals with HIV infection as well as the general population, particularly in sub-Saharan Africa where more than two thirds 
of the global population of HIV-infected individuals resides [7]. Previous studies in Kenya have reported a lower prevalence of overweight/obesity and hypertension among HIV-infected people than in the general population and in those without HIV infection [8, 9]. However, the situation among Kenyans with HIV infection may have changed since the publication of those reports, as has been the case in South Africa [10,11]. Overweight/ obesity is a known risk factor for NCDs and is an important issue to address in NCD prevention. Yet challenges remain with respect to reducing overweight/ obesity among HIV-infected individuals in African countries, where a plump figure is desirable $[12,13]$ and where AIDS has been thought of as a wasting syndrome [14].

In this study, we aimed to determine the prevalence of overweight/obesity and abdominal obesity as well as hypertension among HIV-infected individuals in Western Kenya and to identify the risk factors of overweight/ obesity, abdominal obesity, and hypertension.

\section{Methods}

\section{Study design}

We conducted a cross-sectional study in Mbita, Homa Bay County, Kenya, from September to December 2015. HIV-infected adults were recruited at Mbita Sub-county Hospital and were administered a structured questionnaire as well as anthropometric (weight, height, and waist circumference) and blood pressure measurement.

\section{Participants and sample size}

HIV-infected adults in this study were defined as individuals aged 18 years and older, who had tested positive for HIV infection, were enrolled at the Patient Support Center (PSC) in Mbita Sub-county Hospital for more than 1 year at the time of the study, and had medical records available that included the results of HIV viral load counts within the previous 18 months. All eligible participants at the PSC on the research day were recruited. We excluded individuals who were pregnant; taking hormonal medication, except for contraceptives; and those who were hospitalized.

The sample size was calculated based on the expected prevalence obtained from a previous study conducted among HIV-infected people in Western Kenya where the overall prevalence of overweight and obesity was $18.3 \%$ in both men and women [9]. We applied the formula of sample size for a prevalence study with a crosssection design [15], with 0.183 as the expected prevalence, $95 \%$ confidence interval (CI) of 1.96, and a margin of error of 0.05 for precision. The calculated sample size was 227 . Considering a $10 \%$ non-response rate, 251 participants were recruited during the study period.

\section{Variables}

The main dependent variables were body mass index (BMI), waist circumference (WC), and blood pressure. Weight was measured to the nearest $0.1 \mathrm{~kg}$, height to the nearest $1 \mathrm{~mm}$, and $\mathrm{WC}$ to the nearest $1 \mathrm{~cm}$. Blood pressure was measured once using a digital sphygmomanometer (HEM-7132; OMRON, Kyoto, Japan). BMI $\leq 18.5$ was classified as underweight, $\mathrm{BMI} \geq 25$ as overweight, and $\mathrm{BMI} \geq 30$ as obese [16]. For the analysis, overweight and obesity were combined for outcome and designated as overweight. Abdominal obesity was defined as WC $\geq$ $94 \mathrm{~cm}$ for men and $\geq 80 \mathrm{~cm}$ for women [17]. The definition of hypertension followed the World Health Organization (WHO) criteria, with diastolic pressure $\geq$ $90 \mathrm{mmHg}$ or systolic pressure $\geq 140 \mathrm{mmHg}$ [18].

Independent variables were collected using a questionnaire and medical records. The questionnaire comprised demographic information including socioeconomic status (SES), dietary habits, physical activity, and perception of body size in relation to HIV/AIDS. Alcohol use and smoking were also queried (yes or no) in the questionnaire. The following clinical variables were obtained from the medical records: WHO clinical stage of HIV/ AIDS [19], intake and duration of ART and protease inhibitors (PIs), CD4 counts, viral load, history of opportunistic infections, and history of the use of tuberculosis medication and contraceptives. We collected information of any type of opportunistic infection noted by a clinician in participants' medical records.

In the analysis, education level was recategorized as follows: "No education" and "Incomplete primary school" were recategorized into "low education," "Complete primary school" was retained, and "Complete secondary school" and "higher education" were recategorized into "Complete secondary or higher." Occupation was also recategorized according to level of the physical activity as follows. "Not working," "Housework," and "Student" were recategorized into "At home"; "Farmer" and "Fisherman" into "Working outside"; and "Office work," "Engineer," "Small business entrepreneur," and "Tailor" into "Working inside." Other occupations including driver and carpenter were retained as "others." An SES indicator was generated by summing asset ownership, after principal component analysis was applied to weight each asset (car, refrigerator, television, iron, mobile phone, radio, bicycle, sofa, livestock, and poultry). This was divided into four categories according to 25th, 50th, and 75th percentiles and labeled in order from "poorest," "poor," "less poor," to "rich."

The questionnaire was translated into the local language, Dholuo, then back-translated into English to check for consistency. The questionnaire was modified as required after pre-testing among 40 people who were eligible for participation in the study. 
Table 1 Participant characteristics

\begin{tabular}{|c|c|c|c|c|c|c|c|}
\hline & & Number & Percentage & & & Number & Percentage \\
\hline \multirow[t]{5}{*}{$\overline{\text { Age }}$} & $18-19$ & 1 & 0.4 & Socioeconomic status & Poorest & 63 & 25.1 \\
\hline & $20-29$ & 32 & 12.7 & & Poor & 62 & 24.7 \\
\hline & $30-39$ & 104 & 41.4 & & Less poor & 62 & 24.7 \\
\hline & 40 and older & 114 & 45.4 & & Rich & 63 & 25.1 \\
\hline & & & & & Missing data & 1 & \\
\hline \multirow[t]{2}{*}{ Sex } & Male & 82 & 32.7 & Alcohol & No & 223 & 86.7 \\
\hline & Female & 169 & 67.3 & & & & \\
\hline \multirow[t]{6}{*}{ Education level } & No education & 11 & 4.4 & & Yes & 24 & 9.7 \\
\hline & & & & & Missing data & 4 & \\
\hline & Low education & 85 & 33.9 & Smoke & No & 242 & 96.4 \\
\hline & Complete primary & 117 & 46.6 & & & & \\
\hline & Complete secondary & 30 & 12.0 & & Yes & 9 & 3.6 \\
\hline & Higher education & 8 & 3.2 & Contraceptive use & No & 71 & 28.3 \\
\hline \multirow[t]{10}{*}{ Occupation } & Not working & 26 & 10.4 & & Yes & 180 & 71.7 \\
\hline & Housework & 5 & 2.0 & & Missing data & 4 & \\
\hline & Farmer & 17 & 6.8 & Opportunistic infection history & No & 204 & 81.9 \\
\hline & Fisherman & 31 & 12.4 & & & & \\
\hline & Office work & 3 & 1.2 & & Yes & 45 & 18.1 \\
\hline & Engineer & 1 & 0.4 & & Missing data & 2 & \\
\hline & Small entrepreneur & 125 & 49.8 & Tuberculosis history & No & 66 & 26.3 \\
\hline & Tailor & 7 & 2.8 & & Yes & 185 & 73.7 \\
\hline & Student & 0 & 0.0 & CD4 counts (cells/ $\mu \mathrm{l})$ & Less than 200 & 30 & 12.1 \\
\hline & Other & 36 & 14.3 & & & & \\
\hline \multirow[t]{3}{*}{ On anti-retroviral therapy (ART) } & No & 0 & 0.0 & & & & \\
\hline & Yes & 251 & 100.0 & & $200-499$ & 113 & 45.4 \\
\hline & & & & & 500 and higher & 106 & 42.6 \\
\hline \multirow[t]{5}{*}{ ART duration } & $0-14$ days & 0 & 0.0 & & Missing data & 2 & \\
\hline & $14-55$ days & 1 & 0.4 & Viral load (copies/ml) & Less than 150 & 206 & 82.4 \\
\hline & 56-181 days & 0 & 0.0 & & & & \\
\hline & 182-364 days & 13 & 5.2 & & 150 and higher & 44 & 17.6 \\
\hline & 365 days and more & 237 & 94.4 & & Missing data & 1 & \\
\hline \multirow[t]{3}{*}{ On protease inhibitors } & No & 214 & 85.3 & WHO stage & 1 & 51 & 20.3 \\
\hline & Yes & 36 & 14.3 & & 2 & 71 & 28.3 \\
\hline & Missing data & 1 & & & 3 & 97 & 38.6 \\
\hline \multirow[t]{3}{*}{ Protease inhibitor duration } & $0-539$ days & 7 & 2.8 & & 4 & 29 & 11.6 \\
\hline & 540 days and more & 28 & 11.2 & & Missing data & 3 & \\
\hline & Missing data & 1 & & & & & \\
\hline
\end{tabular}

$N$ number of study participants, $n$ number of elements in a sample. Alcohol use and smoking were also asked as yes or no in the questionnaire. Contraceptive use: hormonal contraceptive use among women. WHO stage: WHO clinical staging of HIV/AIDS for adults and adolescents

\section{Physical activity level}

Physical activity level during the previous 7 days was assessed using the self-administered International Physical Activity Questionnaire for young and middle-aged adults [20]. This instrument has been validated in many countries, including South Africa [20, 21]. Physical activity was categorized into three levels: inactive, minimally active, and health-enhancing physical activity (HEPA).

\section{Perception of body size (body image)}

Participants' perception of body size in relation to HIV/ AIDS was assessed using nine illustrations of different 
body figures, which was presented by Lynch et al. in 2009 [22].

\section{Statistical analysis}

Descriptive analysis was conducted to determine the prevalence of overweight, abdominal obesity, and hypertension along with stratification by age and sex, and for key characteristics among participants. First, the association of overweight, abdominal obesity, and hypertension with each independent variable was examined using bivariate analysis by calculating the crude odds ratio (OR) and 95\% CI. Then, the OR of each variable was adjusted by age, sex, and SES in multivariable logistic regression analysis to identify the adjusted odds ratio (aOR) and 95\% CI. The statistical significance was set as $P$ value $=0.05$. The statistical analysis was conducted using MedCalc version 19.1.7.

\section{Results}

Background data of participants

A total 251 participants were enrolled from among those registered at PSC in Mbita Sub-county Hospital. As the total number of those registered at this hospital as of September to December in 2015 was unavailable, it was difficult to determine the response rate. Participants' characteristics are shown in Table 1. The median age was 38 years with an interquartile range of 32-45 years. All participants had taken ART, most for 1 year or longer. Approximately $75 \%$ of participants had a history of tuberculosis, and fewer than $20 \%$ had a past history of other opportunistic infections. Viral load was less than 150 copies/ml in approximately $80 \%$ of participants.

\section{Prevalence of overweight, abdominal obesity, and hypertension}

More HIV-infected women were overweight than underweight; only $8.3 \%$ of HIV-infected women were underweight whereas $17.2 \%$ were overweight and $3.6 \%$ were obese. Among HIV-infected men, slightly more were underweight (12.2\%) than overweight (11.0\%), and none were obese (data not shown in the table).

The prevalence of overweight, abdominal obesity, and hypertension according to different sex and age groups is shown in Table 2. The prevalence of overweight was higher at age 30 years and older. Abdominal obesity was much more common in women $(62.1 \%)$ than in men (9.6\%), with nearly $70 \%$ of women aged $30-39$ years having abdominal obesity. The prevalence of hypertension in all age groups was $9.8 \%$ and $11.8 \%$ among men and women, respectively. Men tended to develop hypertension at early ages.
Table 2 Prevalence of overweight, abdominal obesity, and hypertension according to sex and age group

\begin{tabular}{|c|c|c|c|c|c|c|c|c|c|}
\hline \multirow[b]{2}{*}{ Age } & \multicolumn{3}{|c|}{ Overweight } & \multicolumn{3}{|c|}{ Abdominal obesity } & \multicolumn{3}{|c|}{ Hypertension } \\
\hline & $N$ & $n$ & $\%$ & $N$ & $n$ & $\%$ & $N$ & $n$ & $\%$ \\
\hline \multicolumn{10}{|l|}{ Male } \\
\hline All ages & 82 & 9 & 11.0 & 82 & 7 & 8.5 & 82 & 8 & 9.8 \\
\hline $18-19$ & 0 & 0 & 0 & 0 & 0 & 0.0 & 0 & 0 & 0.0 \\
\hline $20-29$ & 7 & 0 & 0 & 7 & 1 & 14.3 & 7 & 1 & 14.3 \\
\hline $30-39$ & 28 & 3 & 10.7 & 34 & 2 & 5.9 & 28 & 4 & 14.3 \\
\hline 40 and older & 47 & 6 & 12.8 & 41 & 5 & 12.2 & 47 & 3 & 6.4 \\
\hline \multicolumn{10}{|l|}{ Female } \\
\hline All ages & 169 & 35 & 20.7 & 169 & 105 & 62.1 & 169 & 20 & 11.8 \\
\hline $18-19$ & 1 & 0 & 0 & 1 & 0 & 0.0 & 1 & 0 & 0 \\
\hline $20-29$ & 25 & 3 & 12.0 & 25 & 15 & 60 & 25 & 2 & 8.0 \\
\hline 30-39 & 76 & 17 & 22.4 & 70 & 48 & 68.6 & 76 & 4 & 5.3 \\
\hline 40 and older & 67 & 15 & 22.4 & 73 & 42 & 57.5 & 67 & 14 & 20.9 \\
\hline
\end{tabular}

Overweight includes obesity. Hypertension includes both high systolic blood pressure and diastolic blood pressure. $N$ number of study participants, $n$ number of elements in a sample

\section{Factors associated with overweight, abdominal obesity, and hypertension}

The results of bivariate and multivariate analysis for overweight are shown in Table 3. A history of opportunistic infections was significantly associated with overweight (OR 2.46, 95\% CI 1.10-5.50, $P=$ 0.028). Overweight was more common in women $(19.5 \%)$ than in men $(11.0 \%)$, although no association was identified.

The results of bivariate and multivariate analysis for abdominal obesity are shown in Table 4 . We identified an association between abdominal obesity and female sex (aOR 15.28, 95\% CI 6.84-34.12, $P<0.0001$ ). Abdominal obesity was more common in participants with a history of opportunistic infections (53.3\%) than in those without this history (43.1\%), although no association was identified. Other factors including level of physical activity was not significantly associated with either overweight or abdominal obesity.

The results of bivariate and multivariate analysis with hypertension are shown in Table 5. We identified an association between hypertension and WHO clinical stage. WHO clinical stage 3 was less strongly associated with hypertension than WHO clinical stage 1 (aOR 0.18, 95\% CI $0.05-0.58, P<0.01$ ), as was the case for WHO clinical stage 4 in comparison with WHO clinical stage 1 (aOR 0.16, 95\% CI 0.02-0.87, $P<0.05$ ).

\section{Perceptions of body size}

More than half of HIV-infected participants $(n=$ $150 ; 60 \%$ ) assumed that the thinnest among the nine body figures was indicative of HIV infection whereas 
Table 3 Factors associated with overweight

\begin{tabular}{|c|c|c|c|c|c|c|c|c|c|c|c|}
\hline \multirow{2}{*}{ Age category } & \multirow[t]{2}{*}{$N$} & \multirow[t]{2}{*}{$n$} & \multirow[t]{2}{*}{$\%$} & \multirow[t]{2}{*}{$\mathrm{OR}$} & \multicolumn{2}{|c|}{$95 \% \mathrm{Cl}$} & \multirow[t]{2}{*}{$P$ value } & \multirow[t]{2}{*}{$\mathrm{aOR}$} & \multicolumn{2}{|c|}{$95 \% \mathrm{Cl}$} & \multirow[t]{2}{*}{$P$ value } \\
\hline & & & & & & & & & & & \\
\hline $18-29$ & 33 & 4 & 12.1 & 1.00 & & & & 1.00 & & & \\
\hline $30-39$ & 104 & 20 & 19.2 & 1.72 & 0.54 & 5.47 & 0.353 & 1.99 & 0.61 & 6.46 & 0.251 \\
\hline 40 and older & 114 & 18 & 15.8 & 1.35 & 0.42 & 4.33 & 0.604 & 1.43 & 0.44 & 4.66 & 0.545 \\
\hline \multicolumn{12}{|l|}{ Sex } \\
\hline Male & 82 & 9 & 11.0 & 1.00 & & & & 1.00 & & & \\
\hline Female & 169 & 33 & 19.5 & 1.96 & 0.89 & 4.33 & 0.093 & 2.11 & 0.94 & 4.72 & 0.070 \\
\hline \multicolumn{12}{|l|}{ Marital status } \\
\hline Single & 7 & 2 & 28.6 & 1.00 & & & & 1.00 & & & \\
\hline Married & 154 & 25 & 16.2 & 0.48 & 0.08 & 2.63 & 0.402 & 0.53 & 0.09 & 3.05 & 0.478 \\
\hline Divorced & 10 & 1 & 10.0 & 0.27 & 0.01 & 3.88 & 0.341 & 0.38 & 0.02 & 5.69 & 0.484 \\
\hline Widowed & 80 & 14 & 17.5 & 0.53 & 0.09 & 3.02 & 0.474 & 0.58 & 0.09 & 3.51 & 0.558 \\
\hline \multicolumn{12}{|l|}{ Education level } \\
\hline Low education & 96 & 14 & 14.6 & 1.00 & & & & 1.00 & & & \\
\hline Complete primary & 117 & 22 & 18.8 & 1.35 & 0.65 & 2.82 & 0.414 & 1.59 & 0.74 & 3.44 & 0.229 \\
\hline Complete secondary or higher & 38 & 6 & 15.8 & 1.09 & 0.38 & 3.11 & 0.859 & 1.59 & 0.50 & 5.04 & 0.429 \\
\hline \multicolumn{12}{|l|}{ Occupation } \\
\hline At home & 31 & 5 & 16.1 & 1.00 & & & & 1.00 & & & \\
\hline Working outside & 48 & 10 & 20.8 & 1.36 & 0.41 & 4.47 & 0.603 & 1.36 & 0.40 & 4.59 & 0.620 \\
\hline Working inside & 136 & 20 & 14.7 & 0.89 & 0.31 & 2.61 & 0.841 & 0.83 & 0.27 & 2.47 & 0.738 \\
\hline Others & 36 & 7 & 19.4 & 1.26 & 0.35 & 4.44 & 0.724 & 1.29 & 0.34 & 4.82 & 0.695 \\
\hline \multicolumn{12}{|l|}{ SES } \\
\hline Poorest & 63 & 13 & 20.6 & 1.00 & & & & 1.00 & & & \\
\hline Poor & 62 & 9 & 14.5 & 0.65 & 0.25 & 1.66 & 0.371 & 0.67 & 0.26 & 1.74 & 0.419 \\
\hline Less poor & 62 & 13 & 21.0 & 1.02 & 0.43 & 2.42 & 0.963 & 0.95 & 0.39 & 2.31 & 0.926 \\
\hline Rich & 63 & 7 & 11.1 & 0.48 & 0.17 & 1.30 & 0.1491 & 0.42 & 0.15 & 1.16 & 0.096 \\
\hline \multicolumn{12}{|l|}{ Physical activity level } \\
\hline Inactive & 11 & 2 & 18.2 & 1.00 & & & & 1.00 & & & \\
\hline Minimally active & 22 & 4 & 18.2 & 1.00 & 0.15 & 6.53 & 1 & 0.92 & 0.13 & 6.29 & 0.933 \\
\hline HEPA active & 215 & 36 & 16.7 & 0.91 & 0.18 & 4.36 & 0.9011 & 0.80 & 0.15 & 4.04 & 0.791 \\
\hline \multicolumn{12}{|l|}{ Chai per day } \\
\hline 0-1 cup & 20 & 3 & 15.0 & 1.00 & & & & 1.00 & & & \\
\hline 2 cups & 58 & 11 & 19.0 & 1.32 & 0.32 & 5.33 & 0.691 & 1.19 & 0.28 & 4.94 & 0.803 \\
\hline 3 cups or more & 173 & 28 & 16.2 & 1.09 & 0.30 & 3.98 & 0.8913 & 0.96 & 0.25 & 3.63 & 0.963 \\
\hline \multicolumn{12}{|l|}{ Soda per week } \\
\hline None & 126 & 23 & 18.3 & 1.00 & & & & 1.00 & & & \\
\hline Once & 64 & 7 & 10.9 & 0.55 & 0.22 & 1.36 & 0.196 & 0.49 & 0.19 & 1.25 & 0.141 \\
\hline Twice or more & 61 & 12 & 19.7 & 1.10 & 0.50 & 2.38 & 0.8157 & 0.99 & 0.44 & 2.21 & 0.984 \\
\hline \multicolumn{12}{|l|}{ Ugali per day } \\
\hline Quarter a plate & 185 & 32 & 17.3 & 1.00 & & & & 1.00 & & & \\
\hline Half a plate & 55 & 8 & 14.5 & 0.81 & 0.35 & 1.88 & 0.631 & 0.97 & 0.40 & 2.31 & 0.946 \\
\hline 3 quarters a plate or more & 8 & 1 & 12.5 & 0.68 & 0.08 & 5.74 & 0.7257 & 0.74 & 0.08 & 6.60 & 0.789 \\
\hline \multicolumn{12}{|l|}{ Alcohol } \\
\hline No & 223 & 35 & 15.7 & 1.00 & & & & 1.00 & & & \\
\hline
\end{tabular}


Table 3 Factors associated with overweight (Continued)

\begin{tabular}{|c|c|c|c|c|c|c|c|c|c|c|c|}
\hline \multirow{2}{*}{ Yes } & \multirow{2}{*}{$\frac{N}{24}$} & \multirow{2}{*}{$\frac{n}{5}$} & \multirow{2}{*}{$\frac{\%}{20.8}$} & \multirow{2}{*}{$\frac{\mathrm{OR}}{1.41}$} & \multicolumn{2}{|c|}{$95 \% \mathrm{Cl}$} & \multirow{2}{*}{$\frac{P \text { value }}{0.5179}$} & \multirow{2}{*}{$\frac{\mathrm{aOR}}{1.66}$} & \multicolumn{2}{|c|}{$95 \% \mathrm{Cl}$} & \multirow{2}{*}{$\frac{P \text { value }}{0.367}$} \\
\hline & & & & & 0.49 & 4.03 & & & 0.54 & 5.04 & \\
\hline \multicolumn{12}{|l|}{ Smoke } \\
\hline No & 242 & 41 & 16.9 & 1.00 & & & & 1.00 & & & \\
\hline Yes & 9 & 1 & 11.1 & 0.61 & 0.07 & 5.03 & 0.648 & 0.91 & 0.10 & 8.20 & 0.935 \\
\hline \multicolumn{12}{|l|}{ Abdominal obesity } \\
\hline No & 138 & 21 & 15.2 & 1.00 & & & & 1.00 & & & \\
\hline Yes & 113 & 21 & 18.6 & 1.27 & 0.65 & 2.46 & 0.477 & 0.94 & 0.43 & 2.03 & 0.885 \\
\hline \multicolumn{12}{|l|}{ Hypertension } \\
\hline No & 222 & 35 & 15.8 & 1.00 & & & & 1.00 & & & \\
\hline Yes & 28 & 7 & 25.0 & 1.78 & 0.70 & 4.51 & 0.223 & 2.04 & 0.77 & 5.39 & 0.147 \\
\hline \multicolumn{12}{|l|}{ ART duration } \\
\hline $0-364$ days & 14 & 4 & 28.6 & 1.00 & & & & 1.00 & & & \\
\hline 365 days and more & 237 & 38 & 16.0 & 0.48 & 0.14 & 1.60 & 0.231 & 0.52 & 0.15 & 1.84 & 0.314 \\
\hline \multicolumn{12}{|l|}{ CD4 counts (cells/ul) } \\
\hline$<200$ & 30 & 3 & 10.0 & 1.00 & & & & 1.00 & & & \\
\hline $200-499$ & 113 & 20 & 17.7 & 1.93 & 0.53 & 7.01 & 0.315 & 2.08 & 0.55 & 7.79 & 0.276 \\
\hline$\geq 500$ & 106 & 19 & 17.9 & 1.96 & 0.54 & 7.15 & 0.3053 & 1.94 & 0.51 & 7.31 & 0.326 \\
\hline \multicolumn{12}{|l|}{ On PI } \\
\hline No & 214 & 39 & 18.2 & 1.00 & & & & 1.00 & & & \\
\hline Yes & 36 & 2 & 5.6 & 0.41 & 0.11 & 1.39 & 0.1537 & 0.45 & 0.12 & 1.57 & 0.323 \\
\hline \multicolumn{12}{|l|}{ PI duration } \\
\hline 0-359 days & 7 & 1 & 14.3 & 1.00 & & & & 1.00 & & & \\
\hline 540 days and more & 28 & 2 & 7.1 & 0.46 & 0.03 & 5.96 & 0.553 & & & & 0.999 \\
\hline \multicolumn{12}{|l|}{ WHO stage } \\
\hline 1 & 51 & 8 & 15.7 & 1.00 & & & & 1.00 & & & \\
\hline 2 & 71 & 17 & 23.9 & 1.69 & 0.66 & 4.29 & 0.268 & 1.85 & 0.71 & 4.86 & 0.208 \\
\hline 3 & 97 & 12 & 12.4 & 0.75 & 0.28 & 1.99 & 0.576 & 0.74 & 0.27 & 2.02 & 0.563 \\
\hline 4 & 29 & 5 & 17.2 & 1.11 & 0.32 & 3.81 & 0.8562 & 1.04 & 0.28 & 3.79 & 0.946 \\
\hline \multicolumn{12}{|l|}{ Ol history } \\
\hline No & 204 & 30 & 14.7 & 1.00 & & & & 1.00 & & & \\
\hline Yes & 45 & 12 & 26.7 & 2.11 & 0.98 & 4.53 & 0.0562 & 2.46 & 1.10 & 5.50 & 0.028 \\
\hline
\end{tabular}

$N$ number of study participants, $n$ number of elements in a sample, OR odds ratio, $95 \% \mathrm{Cl} 95 \%$ confidential interval, $a O R$ adjusted odds ratio, $P$ value probability value, HEPA health-enhancing physical activity, ART anti-retroviral therapy, PI protease inhibitor, WHO stage WHO clinical staging of HIV/AIDS for adults and adolescent, $\mathrm{Ol}$ opportunistic infection

$37.6 \%(n=94)$ did not make this assumption for any of the body figures. Only $1.6 \%(n=4)$ assumed that the largest figure was indicative of HIV infection. Perception of a thin body figure as indicating HIV infection was significantly associated with personal experience of or witnessing discrimination against HIV-infected people (OR 3.67, 95\% CI 2.365.69, $P<0.001)$. However, no association was found between an experience of discrimination and overweight (OR 1.84, 95\% CI 0.94-3.16, $P=0.070$ ) or experience of discrimination and abdominal obesity (OR 1.25, 95\% CI 0.74-2.12, $P=0.398$ ).

\section{Discussion}

The findings of this study highlight the current situation regarding body weight and blood pressure among HIVinfected individuals in Western Kenya.

Overweight was much more prevalent than underweight among women in this study. Moreover, we observed a high prevalence of abdominal obesity among women. First, the second regimen of ART, or PIs, is known to cause metabolic side effects including abdominal obesity [23]; however, only a few individuals in our study population had taken PIs. A high prevalence of abdominal obesity among HIV-infected women who were 
Table 4 Factors associated with abdominal obesity

\begin{tabular}{|c|c|c|c|c|c|c|c|c|c|c|c|}
\hline \multirow{2}{*}{ Age category } & \multirow[t]{2}{*}{$N$} & \multirow[t]{2}{*}{$n$} & \multirow[t]{2}{*}{$\%$} & \multirow[t]{2}{*}{ OR } & \multicolumn{2}{|c|}{$95 \% \mathrm{Cl}$} & \multirow[t]{2}{*}{$P$ value } & \multirow[t]{2}{*}{$\mathrm{aOR}$} & \multicolumn{2}{|c|}{$95 \% \mathrm{Cl}$} & \multirow[t]{2}{*}{$P$ value } \\
\hline & & & & & & & & & & & \\
\hline $18-29$ & 33 & 16 & 48.5 & 1.00 & & & & 1.00 & & & \\
\hline $30-39$ & 104 & 50 & 48.1 & 0.98 & 0.44 & 2.15 & 0.967 & 1.30 & 0.53 & 3.19 & 0.558 \\
\hline 40 and older & 114 & 47 & 41.2 & 0.74 & 0.34 & 1.66 & 0.459 & 1.06 & 0.44 & 22.54 & 0.896 \\
\hline \multicolumn{12}{|l|}{ Sex } \\
\hline Male & 82 & 8 & 9.8 & 1.00 & & & & 1.00 & & & \\
\hline Female & 169 & 105 & 62.1 & 15.17 & 6.86 & 33.53 & $<0.0001$ & 15.28 & 6.84 & 34.12 & $<0.0001$ \\
\hline \multicolumn{12}{|l|}{ Marital status } \\
\hline Single & 7 & 3 & 42.9 & 1 & & & & 1 & & & \\
\hline Married & 154 & 71 & 46.1 & 1.14 & 0.24 & 5.26 & 0.866 & 1.94 & 0.35 & 10.57 & 0.441 \\
\hline Divorced & 10 & 4 & 40.0 & 0.88 & 0.12 & 6.31 & 0.906 & 2.11 & 0.21 & 20.42 & 0.516 \\
\hline Widowed & 80 & 35 & 43.8 & 1.03 & 0.21 & 4.93 & 0.963 & 2.18 & 0.37 & 12.68 & 0.382 \\
\hline \multicolumn{12}{|l|}{ Education level } \\
\hline Low education & 96 & 41 & 42.7 & 1.00 & & & & 1.00 & & & \\
\hline Complete primary & 117 & 51 & 43.6 & 1.03 & 0.60 & 1.78 & 0.897 & 0.86 & 0.45 & 1.67 & 0.676 \\
\hline Complete secondary or higher & 38 & 21 & 55.3 & 1.65 & 0.77 & 3.53 & 0.19 & 1.34 & 0.50 & 3.61 & 0.551 \\
\hline \multicolumn{12}{|l|}{ Occupation } \\
\hline At home & 31 & 15 & 48.4 & 1.00 & & & & 1.00 & & & \\
\hline Working outside & 48 & 23 & 47.9 & 0.98 & 0.39 & 2.42 & 0.967 & 0.79 & 0.26 & 2.40 & 0.685 \\
\hline Working inside & 136 & 57 & 41.9 & 0.76 & 0.35 & 1.68 & 0.511 & 0.63 & 0.24 & 1.67 & 0.360 \\
\hline Others & 36 & 18 & 50.0 & 1.06 & 0.40 & 2.78 & 0.895 & 0.57 & 0.17 & 1.85 & 0.357 \\
\hline \multicolumn{12}{|l|}{ SES } \\
\hline Poorest & 63 & 23 & 36.5 & 1.00 & & & & 1.00 & & & \\
\hline Poor & 62 & 23 & 37.1 & 1.02 & 0.49 & 2.12 & 0.945 & 0.99 & 0.43 & 2.28 & 0.990 \\
\hline Less poor & 62 & 32 & 51.6 & 1.85 & 0.90 & 3.79 & 0.090 & 1.54 & 0.68 & 3.51 & 0.294 \\
\hline Rich & 63 & 35 & 55.6 & 2.17 & 1.06 & 4.44 & 0.033 & 1.99 & 0.86 & 4.60 & 0.104 \\
\hline \multicolumn{12}{|l|}{ Physical activity level } \\
\hline Inactive & 11 & 6 & 54.5 & 1.00 & & & & & & & \\
\hline Minimally active & 22 & 9 & 40.9 & 0.57 & 0.13 & 2.48 & 0.46 & 0.40 & 0.07 & 2.28 & 0.302 \\
\hline HEPA active & 215 & 97 & 45.1 & 0.68 & 0.20 & 2.31 & 0.542 & 0.55 & 0.12 & 2.40 & 0.429 \\
\hline \multicolumn{12}{|l|}{ Chai per day } \\
\hline 0-1 cup & 20 & 8 & 40.0 & 1.00 & & & & 1.00 & & & \\
\hline 2 cups & 58 & 27 & 46.6 & 1.30 & 0.46 & 3.66 & 0.611 & 1.14 & 0.32 & 4.05 & 0.836 \\
\hline
\end{tabular}

not taking PIs is compatible with the findings of a previous study reporting a positive association between abdominal obesity and HIV infection with an ART regimen, even with minor metabolic side effects [24]. Second, abdominal obesity among women was defined as $\mathrm{WC} \geq 80 \mathrm{~cm}$ [17]; however, this cutoff point is controversial [25-29]. The WC cutoff point for abdominal obesity among sub-Saharan African populations is based on the cutoff point obtained from studies conducted among European populations [17]. A study targeting HIV-infected people in South Africa determined that 90 $\mathrm{cm}$ was an optimal cutoff point [29]. Furthermore, the combined use of WC and hip circumference could more effectively predict an increased health risk among HIVinfected individuals than use of WC alone [30]. Therefore, further investigation using different cutoff points and measurement methods is desirable.

Only female sex was found to be a risk factor of abdominal obesity, which is in agreement with a previous study [24]. In our study, no other potential associations were found for other dependent variables such as high SES, being married, and older age, as have been reported in previous studies $[10,31]$. A possible reason for this might be bias and a small study population with similar 
Table 4 Factors associated with abdominal obesity (Continued)

\begin{tabular}{|c|c|c|c|c|c|c|c|c|c|c|c|}
\hline \multirow{2}{*}{3 cups or more } & \multirow{2}{*}{$\frac{N}{173}$} & \multirow{2}{*}{$\frac{n}{78}$} & \multirow{2}{*}{$\frac{\%}{45.1}$} & \multirow{2}{*}{$\frac{\mathrm{OR}}{1.23}$} & \multicolumn{2}{|c|}{$95 \% \mathrm{Cl}$} & \multirow{2}{*}{$\frac{P \text { value }}{0.665}$} & \multirow{2}{*}{$\frac{\mathrm{aOR}}{0.87}$} & \multicolumn{2}{|c|}{$95 \% \mathrm{Cl}$} & \multirow{2}{*}{$\frac{P \text { value }}{0.827}$} \\
\hline & & & & & 0.47 & 3.16 & & & 0.27 & 2.79 & \\
\hline \multicolumn{12}{|l|}{ Soda per week } \\
\hline None & 126 & 58 & 46.0 & 1.00 & & & & 1.00 & & & \\
\hline Once & 64 & 27 & 42.2 & 0.85 & 0.46 & 1.57 & 0.614 & 0.66 & 0.32 & 1.34 & 0.255 \\
\hline Twice or more & 61 & 28 & 45.9 & 0.99 & 0.53 & 1.83 & 0.986 & 0.96 & 0.46 & 2.03 & 0.933 \\
\hline \multicolumn{12}{|l|}{ Ugali per day } \\
\hline Quarter a plate & 185 & 91 & 49.2 & 1.00 & & & & 1.00 & & & \\
\hline Half a plate & 55 & 19 & 34.5 & 0.54 & 0.29 & 1.01 & 0.057 & 0.60 & 0.28 & 1.27 & 0.186 \\
\hline 3 quarters a plate or more & 8 & 2 & 25.0 & 0.34 & 0.06 & 1.75 & 0.198 & 0.36 & 0.04 & 1.27 & 0.205 \\
\hline \multicolumn{12}{|l|}{ Alcohol } \\
\hline No & 223 & 103 & 46.2 & 1.00 & & & & 1.00 & & & \\
\hline Yes & 24 & 8 & 33.3 & 0.58 & 0.23 & 1.41 & 0.233 & 1.16 & 0.38 & 3.52 & 0.792 \\
\hline \multicolumn{12}{|l|}{ Smoke } \\
\hline No & 242 & 112 & 46.3 & 1.00 & & & & 1.00 & & & \\
\hline Yes & 9 & 1 & 11.1 & 0.14 & 0.01 & 1.17 & 0.07 & 0.52 & 0.04 & 5.54 & 0.590 \\
\hline \multicolumn{12}{|l|}{ Abdominal obesity } \\
\hline No & 209 & 92 & 44.0 & 1.00 & & & & 1.00 & & & \\
\hline Yes & 42 & 21 & 50.0 & 1.27 & 0.65 & 2.46 & 0.477 & 0.95 & 0.44 & 2.09 & 0.900 \\
\hline \multicolumn{12}{|l|}{ Hypertension } \\
\hline No & 222 & 97 & 43.7 & 1.00 & & & & 1.00 & & & \\
\hline Yes & 28 & 16 & 57.1 & 1.71 & 0.77 & 3.80 & 0.181 & 1.72 & 0.65 & 4.53 & 0.272 \\
\hline \multicolumn{12}{|l|}{ ART duration } \\
\hline 0-364 days & 14 & 9 & 64.3 & 1.00 & & & & 1.00 & & & \\
\hline 365 days and more & 237 & 104 & 43.9 & 0.43 & 0.14 & 1.33 & 0.145 & 0.34 & 0.08 & 1.39 & 0.136 \\
\hline \multicolumn{12}{|l|}{  } \\
\hline$<200$ & 30 & 15 & 50.0 & 1.00 & & & & 1.00 & & & \\
\hline $200-499$ & 113 & 50 & 44.2 & 0.79 & 0.35 & 1.77 & 0.574 & 1.04 & 0.41 & 2.65 & 0.927 \\
\hline$\geq 500$ & 106 & 48 & 45.3 & 0.82 & 0.36 & 1.86 & 0.647 & 1.07 & 0.41 & 2.75 & 0.885 \\
\hline \multicolumn{12}{|l|}{ On PI } \\
\hline No & 214 & 95 & 44.4 & 1.00 & & & & 1.00 & & & \\
\hline Yes & 36 & 18 & 50.0 & 1.25 & 0.61 & 2.53 & 0.532 & 1.82 & 0.74 & 4.44 & 0.187 \\
\hline \multicolumn{12}{|l|}{ PI duration } \\
\hline 0-359 days & 7 & 3 & 42.9 & 1.00 & & & & 1.00 & & & \\
\hline 540 days and more & 28 & 15 & 53.6 & 1.53 & 0.28 & 8.18 & 0.613 & 0.04 & 0.00 & 2.60 & 0.131 \\
\hline \multicolumn{12}{|l|}{ WHO stage } \\
\hline 1 & 51 & 20 & 39.2 & 1.00 & & & & 1.00 & & & \\
\hline 2 & 71 & 37 & 52.1 & 1.68 & 0.81 & 3.50 & 0.160 & 2.37 & 0.97 & 5.73 & 0.056 \\
\hline 3 & 97 & 40 & 41.2 & 1.08 & 0.54 & 2.17 & 0.811 & 1.14 & 0.51 & 2.58 & 0.736 \\
\hline 4 & 29 & 15 & 51.7 & 1.66 & 0.66 & 4.16 & 0.279 & 1.85 & 0.61 & 5.57 & 0.269 \\
\hline \multicolumn{12}{|l|}{ Ol history } \\
\hline No & 204 & 88 & 43.1 & 1.00 & & & & 1.000 & & & \\
\hline Yes & 45 & 24 & 53.3 & 1.50 & 0.78 & 2.87 & 0.215 & 2.21 & 0.97 & 5.02 & 0.057 \\
\hline
\end{tabular}

$N$ number of study participants, $n$ number of elements in a sample, OR odds ratio, $95 \% \mathrm{Cl} 95 \%$ confidential interval, aOR adjusted odds ratio, $P$ value probability value, HEPA health-enhancing physical activity, ART anti-retroviral therapy, PI protease inhibitor, WHO stage WHO clinical staging of HIV/AIDS for adults and adolescents, $\mathrm{Ol}$ opportunistic infection 
Table 5 Factors associated with hypertension

\begin{tabular}{|c|c|c|c|c|c|c|c|c|c|c|c|}
\hline \multirow{2}{*}{ Age category } & \multirow[t]{2}{*}{$N$} & \multirow[t]{2}{*}{$n$} & \multirow[t]{2}{*}{$\%$} & \multirow[t]{2}{*}{ OR } & \multicolumn{2}{|c|}{$95 \% \mathrm{Cl}$} & \multirow[t]{2}{*}{$P$ value } & \multirow[t]{2}{*}{$\mathrm{aOR}$} & \multicolumn{2}{|c|}{$95 \% \mathrm{Cl}$} & \multirow[t]{2}{*}{$P$ value } \\
\hline & & & & & & & & & & & \\
\hline $18-29$ & 33 & 3 & 9.1 & 1.00 & & & & 1.00 & & & \\
\hline $30-39$ & 104 & 8 & 7.7 & 0.83 & 0.21 & 3.34 & 0.797 & 0.75 & 0.18 & 3.08 & 0.308 \\
\hline 40 and older & 113 & 17 & 15.0 & 1.77 & 0.48 & 6.45 & 0.387 & 1.93 & 0.51 & 7.23 & 0.952 \\
\hline \multicolumn{12}{|l|}{ Sex } \\
\hline Male & 81 & 8 & 9.9 & 1.00 & & & & 1.00 & & & \\
\hline Female & 169 & 20 & 11.8 & 1.22 & 0.51 & 2.91 & 0.646 & 1.23 & 0.51 & 2.99 & 0.645 \\
\hline \multicolumn{12}{|l|}{ Marital status } \\
\hline Single & 7 & 0 & 0.0 & 1.00 & & & & 1.00 & & & \\
\hline Married & 154 & 17 & 11.0 & & & & 0.999 & & & & 0.999 \\
\hline Divorced & 10 & 0 & 0.0 & 1.00 & & & 1.000 & & & & 1.000 \\
\hline Widowed & 79 & 11 & 13.9 & 1.00 & & & 0.999 & & & & 0.999 \\
\hline \multicolumn{12}{|l|}{ Education level } \\
\hline Low education & 96 & 13 & 13.5 & 1.00 & & & & 1.00 & & & \\
\hline Complete primary & 116 & 11 & 9.5 & 0.66 & 0.28 & 1.56 & 0.355 & 0.59 & 0.23 & 1.47 & 0.260 \\
\hline Complete secondary or higher & 38 & 4 & 10.5 & 0.75 & 0.22 & 2.46 & 0.637 & 0.42 & 0.11 & 1.71 & 0.229 \\
\hline \multicolumn{12}{|l|}{ Occupation } \\
\hline At home & 31 & 2 & 6.5 & 1.00 & & & & 1.00 & & & \\
\hline Working outside & 48 & 4 & 8.3 & 1.31 & 0.22 & 7.66 & 0.759 & 1.35 & 0.22 & 8.11 & 0.741 \\
\hline Working inside & 135 & 17 & 12.6 & 2.08 & 0.45 & 9.55 & 0.342 & 1.98 & 0.42 & 9.35 & 0.387 \\
\hline Others & 36 & 5 & 13.9 & 2.33 & 0.42 & 13.01 & 0.332 & 1.79 & 0.31 & 10.49 & 0.516 \\
\hline \multicolumn{12}{|l|}{ SES } \\
\hline Poorest & 63 & 7 & 11.1 & 1.00 & & & & 1.00 & & & \\
\hline Poor & 62 & 4 & 6.5 & 0.55 & 0.15 & 1.98 & 0.363 & 0.51 & 0.14 & 1.86 & 0.308 \\
\hline Less poor & 62 & 7 & 11.3 & 1.01 & 0.33 & 3.09 & 0.975 & 0.96 & 0.31 & 2.98 & 0.952 \\
\hline Rich & 62 & 10 & 16.1 & 1.53 & 0.54 & 4.33 & 0.414 & 1.78 & 0.61 & 5.17 & 0.289 \\
\hline \multicolumn{12}{|l|}{ Physical activity level } \\
\hline Inactive & 11 & 2 & 18.2 & 1.00 & & & & 1.00 & & & \\
\hline Minimally active & 22 & 2 & 9.1 & 0.45 & 0.05 & 3.71 & 0.459 & 0.46 & 0.05 & 4.01 & 0.482 \\
\hline HEPA active & 214 & 24 & 11.2 & 0.56 & 0.11 & 2.78 & 0.486 & 0.46 & 0.08 & 2.43 & 0.364 \\
\hline \multicolumn{12}{|l|}{ Chai per day } \\
\hline 0-1 cup & 20 & 0 & 0.0 & 1.00 & & & & 1.00 & & & \\
\hline 2 cups & 58 & 1 & 1.7 & & & & 0.998 & & & & 0.998 \\
\hline 3 cups or more & 172 & 27 & 15.7 & & & & 0.997 & & & & 0.998 \\
\hline \multicolumn{12}{|l|}{ Soda per week } \\
\hline None & 125 & 19 & 15.2 & 1.00 & & & & 1.00 & & & \\
\hline Once & 64 & 4 & 6.3 & 0.37 & 0.12 & 1.14 & 0.085 & 0.41 & 0.13 & 1.29 & 0.130 \\
\hline Twice or more & 61 & 5 & 8.2 & 0.49 & 0.17 & 1.41 & 0.188 & 0.57 & 0.20 & 1.66 & 0.311 \\
\hline \multicolumn{12}{|l|}{ Ugali per day } \\
\hline Quarter a plate & 184 & 24 & 13.0 & 1.00 & & & & 1.00 & & & \\
\hline Half a plate & 55 & 4 & 7.3 & 0.52 & 0.17 & 1.57 & 0.250 & 0.50 & 0.16 & 1.54 & 0.230 \\
\hline 3 quarters a plate or more & 8 & 0 & 0.0 & & & & 0.998 & & & & 0.998 \\
\hline \multicolumn{12}{|l|}{ Alcohol } \\
\hline No & 222 & 24 & 10.8 & 1.00 & & & & 1.00 & & & \\
\hline
\end{tabular}


Table 5 Factors associated with hypertension (Continued)

\begin{tabular}{|c|c|c|c|c|c|c|c|c|c|c|c|}
\hline \multirow[b]{2}{*}{ Yes } & \multirow{2}{*}{$\frac{N}{24}$} & \multirow{2}{*}{$\frac{n}{3}$} & \multirow{2}{*}{$\frac{\%}{12.5}$} & \multirow{2}{*}{$\frac{\mathrm{OR}}{1.17}$} & \multicolumn{2}{|c|}{$95 \% \mathrm{Cl}$} & \multirow{2}{*}{$\frac{P \text { value }}{0.802}$} & \multirow{2}{*}{$\frac{\mathrm{aOR}}{1.35}$} & \multicolumn{2}{|c|}{$95 \% \mathrm{Cl}$} & \multirow{2}{*}{$\frac{P \text { value }}{0.661}$} \\
\hline & & & & & 0.32 & 4.24 & & & 0.35 & 5.23 & \\
\hline \multicolumn{12}{|l|}{ Smoke } \\
\hline No & 241 & 28 & 11.6 & 1.00 & & & & 1.00 & & & \\
\hline Yes & 9 & 0 & 0.0 & & & & 0.998 & & & & 0.998 \\
\hline \multicolumn{12}{|l|}{ Overweight/obesity } \\
\hline No & 208 & 21 & 10.1 & 1.00 & & & & 1.00 & & & \\
\hline Yes & 42 & 7 & 16.7 & 1.78 & 0.70 & 4.51 & 0.223 & 1.95 & 0.74 & 5.13 & 0.173 \\
\hline \multicolumn{12}{|l|}{ Abdominal obesity } \\
\hline No & 137 & 12 & 8.8 & & & & & 1.00 & & & \\
\hline Yes & 113 & 16 & 14.2 & & & & & 1.71 & 0.66 & 4.42 & 0.261 \\
\hline \multicolumn{12}{|l|}{ ART duration } \\
\hline $0-364$ days & 14 & 2 & 14.3 & 1.00 & & & & 1.00 & & & \\
\hline 365 days and more & 236 & 26 & 11.0 & 1.71 & 0.77 & 3.80 & 0.182 & 0.51 & 0.10 & 2.59 & 0.424 \\
\hline \multicolumn{12}{|l|}{ 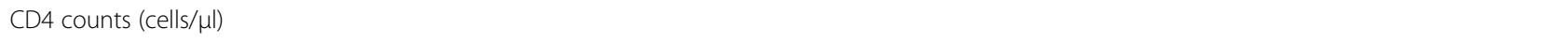 } \\
\hline$<200$ & 30 & 3 & 10.0 & 1.00 & & & & 1.00 & & & \\
\hline $200-499$ & 113 & 13 & 11.5 & 1.17 & 0.31 & 4.40 & 0.816 & 1.51 & 0.38 & 5.95 & 0.555 \\
\hline$\geq 500$ & 105 & 12 & 11.4 & 1.16 & 0.31 & 4.41 & 0.826 & 1.62 & 0.40 & 6.56 & 0.495 \\
\hline \multicolumn{12}{|l|}{ On PI } \\
\hline No & 214 & 26 & 12.1 & 1.00 & & & & 1.00 & & & \\
\hline Yes & 35 & 2 & 5.7 & 0.43 & 0.09 & 1.93 & 0.276 & 0.39 & 0.08 & 1.81 & 0.234 \\
\hline \multicolumn{12}{|l|}{ PI duration } \\
\hline $0-359$ days & 7 & 0 & 0.0 & 1.00 & & & & 1.00 & & & \\
\hline 540 days and more & 27 & 2 & 7.4 & & & & 0.998 & & & & 0.999 \\
\hline \multicolumn{12}{|l|}{ WHO stage } \\
\hline 1 & 51 & 10 & 19.6 & 1.00 & & & & 1.00 & & & \\
\hline 2 & 71 & 10 & 14.1 & 0.67 & 0.25 & 1.75 & 0.414 & 0.52 & 0.18 & 1.44 & 0.211 \\
\hline 3 & 96 & 6 & 6.3 & 0.27 & 0.09 & 0.80 & 0.018 & 0.18 & 0.05 & 0.58 & 0.004 \\
\hline 4 & 29 & 2 & 6.9 & 0.30 & 0.06 & 1.49 & 0.143 & 0.16 & 0.02 & 0.87 & 0.034 \\
\hline \multicolumn{12}{|l|}{ Ol history } \\
\hline No & 204 & 21 & 10.3 & 1.00 & & & & 1.00 & & & \\
\hline Yes & 44 & 7 & 15.9 & 1.64 & 0.65 & 4.15 & 0.290 & 1.52 & 0.57 & 4.00 & 0.396 \\
\hline
\end{tabular}

$N$ number of study participants, $n$ number of elements in a sample, OR odds ratio, $95 \%$ Cl $95 \%$ confidential interval, aOR adjusted odds ratio, $P$ value probability value, HEPA health-enhancing physical activity, ART anti-retroviral therapy, PI protease inhibitor, WHO stage WHO clinical staging of HIV/AIDS for adults and adolescents, Ol opportunistic infection

lifestyles and economic levels. Consumption of sugarsweetened beverages is a well-known risk factor of overweight/obesity in the general population [32]; however, we found no association between consumption of soda or chai and overweigh/obesity in our study population, despite the fact that drinking chai is an integral aspect of Kenyan culture. Physical activity was not found to be associated with a decreased risk of overweight/obesity in this study, despite being widely known as a preventive factor for overweight/obesity and hypertension [33]. Most participants had HEPA levels of physical activity, which implies that people throughout the study area have similar lifestyles. This could explain the lack of an association between chai or soda consumption and overweight/obesity, as high energy consumption was accompanied by high activity levels.

An association of overweight with a history of opportunistic infections was revealed in this study. Studies on the association between overweight/obesity and opportunistic infections are limited, and the precise relationship between them remains unknown. However, a previous report noted that the presence of an opportunistic infection decreased the likelihood of overweight/ obesity [34]. Furthermore, an association between higher 
BMI and higher CD4 counts was reported in a past study [35], which may lessen the likelihood of acquiring an opportunistic infection. However, some evidence of a relationship between obesity and inflammation, such as surgical-site infections, nosocomial infections, periodontitis, and skin infections, has been established [36]. In our study, a causal relationship could not be established owing to the cross-sectional study design. There is a possibility that participants had opportunistic infections at the time of HIV diagnosis or on beginning ART. Then, opportunistic infections were treated and the individual regained weight afterward because ART initiation has been reportedly associated with overweight/obesity [4]. Further research is needed to follow weight change and the occurrence of opportunistic infections or other events, to identify the risk and outcomes of overweight/ obesity.

In our study population, perceptions about body size may remain problematic. Most people associated the thinnest body figure as indicative of HIV infection, having remembered wasting disease during the initial stages of the HIV/AIDS outbreak [14]. This perception might also be reinforced by experiences of discrimination or observing discrimination against people who are thin. In fact, participants who had such experiences were more likely to perceive a thin body figure as indicative of HIV infection.

The findings of this study highlight the necessity of intervention to prevent and decrease overweight and abdominal obesity among people living with HIV as well as among the general population. Our study adds several important points to the knowledge base in this regard. Understanding local beliefs and concepts is important when introducing any new approach to health. Continuous monitoring and further investigation are also necessary because the physical condition of HIV-positive individuals, including their nutritional status, may change according to ART outcome and lifestyle changes.

This study has several limitations. First, we did not include an HIV-negative group for comparison, so it was difficult to identify those factors only affecting people with HIV infection. Second, the data used in this study do not represent the population of the entire study area because the study location and participants were not randomly selected. Third, we failed to recruit a sufficient number of participants to conduct efficient statistical analysis because of the limited study period. Lastly, owing to technical differences and time management issues, we could not measure hip circumference, to obtain the waist-to-hip ratio, which is often used to assess cardiovascular risk in people with HIV infection. Despite these limitations, our study provides informative insights, adding to the knowledge gained in early studies on NCDs and HIV infection on the African continent.

\section{Conclusion}

HIV-infected women were more likely to be overweight or obese than underweight whereas the men with HIV infection in our study tended to be underweight. Abdominal obesity was much common in women, especially among those in their 30s. Female sex and a history of opportunistic infections were identified as risk factors of abdominal obesity and overweight, respectively, and we identified a negative association between hypertension and WHO clinical stage. More than half of participants assumed a thin body figure indicated HIV infection, although none of the assessed factors was significantly associated with this assumption. HIV-infected individuals, especially women, should be targeted in preventive interventions for overweight and abdominal obesity, with consideration of relevant social and cultural aspects. In addition, continuous monitoring and further investigation are necessary as the physical and clinical condition of people living with HIV, including their nutritional status, may change according to ART outcome and lifestyle changes.

\section{Abbreviations}

AIDS: Acquired immune deficiency syndrome; aOR: Adjusted odds ratio; ART: Anti-retroviral therapy; BMI: Body mass index; Cl: Confidence interval; HEPA: Health-enhancing physical activity; HIV: Human immunodeficiency virus; NCDs: Non-communicable diseases; OR: Odds ratio; PIs: Protease inhibitors; PSC: Patient Support Center; SES: Socioeconomic status; WC: Waist circumference; WHO: World Health Organization

\section{Acknowledgements}

We are grateful to the study participants in Mbita Sub-county, Kenya, for their understanding and contribution. We thank Mbita Sub-county Hospital for its support in the study. We acknowledge the valuable support received from the data collectors and their high-quality and patient work. The funder had no role in the preparation of this manuscript in the decision to publish it.

\section{Authors' contributions}

AS, MK, and YK conceived and designed the study. MK supervised the research work in the field. AS have conducted the whole research work and wrote the drafts and revised manuscript. YK thoroughly reviewed and revised the manuscript and checked the references. AS and YK finalized the manuscript. All authors read and approved the final manuscript.

\section{Funding}

This research received partial financial support from our own Institution, School of Tropical Medicine \& Global Health, Nagasaki University. The digital sphygmomanometers (OMRON HEM-7132) and digital scales (OMRON HBF220, Japan) were donated by OMRON Japan.

\section{Availability of data and materials}

The datasets used and analyzed during the current study are available from the corresponding author on reasonable request.

\section{Ethics approval and consent to participate}

The Kenya Medical Research Institute approved the study proposal to be conducted under ongoing research of the Nagasaki University Health Demographic Surveillance System in Suba, Kenya. Approval of the National Committee for Science, Technology and Innovation was then obtained (serial number: A5419). Written informed consent was also obtained in advance from each participant. 


\section{Competing interests}

No potential conflict of interest was reported by the authors.

\section{Author details}

'School of Tropical Medicine \& Global Health, Nagasaki University, 1-12-4, Sakamoto, Nagasaki 852-8523, Japan. ${ }^{2}$ Center for Public Health Research, Kenya Medical Research Institute, Nairobi, Kenya.

Received: 28 December 2019 Accepted: 23 April 2020

Published online: 07 May 2020

\section{References}

1. United Nations. Transforming our World: The 2030 Agenda for Sustainable Development. https://sustainabledevelopment.un.org/content/ documents/21252030\%20Agenda\%20for\%20Sustainable\%2 ODevelopment\%20web.pdf. Accessed 7 Oct. 2019.

2. Europian Environment Agency. Changing diseases burdens and risks of pandemics (GMT 3). https://www.eea.europa.eu/soer-2015/global/health Accessed 7 Oct. 2019.

3. WHO. The top 10 causes of death. https://www.who.int/news-room/factsheets/detail/the-top-10-causes-of-death. Accessed 7 Oct. 2019.

4. Kintu A, Liu E, Hertzmark E, Spiegelman D, Zack RM, Muya A, et al. Incidence and risk factors for overweight and obesity after initiation of antiretroviral therapy in Dar es Salaam, Tanzania. J Int Assoc Provid AIDS Care. 2018. https://doi.org/10.1177/2325958218759759.

5. Terrence Higgins Trust. Uncharted territory: a report into the first generation growing older with HIV. https://www.tht.org.uk/sites/default/files/2018-03/ uncharted_territory_final_low-res.pdf. Publised January 2017. Accessed 7 Oct. 2019.

6. Crum-Cianflone N, Roediger MP, Eberly L, Headd M, Marconi V, Ganesan A Infectious Disease Clinical Research Program HIV Working Group, et al. Increasing rates of obesity among HIV-infected persons during the HIV epidemic. PLoS One, 2010. https://doi.org/10.1371/journal.pone.0010106.

7. Unicef Data. Global and regional trends. https://data.unicef.org/topic/ hivaids/global-regional-trends/. Accessed 8 Oct. 2019.

8. Kuria EN. Food consumption and nutritional status of people living with HIV/AIDS (PLWHA): a case of Thika and Bungoma Districts, Kenya. Public Health Nutr. 2010. https://doi.org/10.1017/S1368980009990826.

9. Bloomfield GS, Hogan JW, Keter A, Sang E, Carter EJ, Velazquez EJ, et al. Hypertension and obesity as cardiovascular risk factors among HIV seropositive patients in Western Kenya. PLoS One. 2011. https://doi.org/10. 1371/journal.pone.0022288.

10. Malaza A, Mossong J, Barnighausen T, Newell ML. Hypertension and obesity in adults living in a high HIV prevalence rural area in South Africa. PLoS One. 2012. https://doi.org/10.1371/journal.pone.0047761.

11. Zhou J, Lurie MN, Barnighausen T, McGarvey ST, Newell ML, Tanser F. Determinants and spatial patterns of adult overweight and hypertension in a high HIV prevalence rural South African population. Health Place. 2012 https://doi.org/10.1016/j.healthplace.2012.09.001.

12. Matoti-Mvalo T, Puoane T. Perceptions of body size and its association with HIV/AIDS. South Afr J Clin Nutr. 2011. https://doi.org/10.1371/journal.pone. 0132914.

13. Puoane T, Fourie JM, Shapiro M, Rosling L, Tshaka NC, Oelefse A. 'Big is beautiful' - an exploration with urban black community health workers in a South African township. South Afr J Clin Nutr. 2005. https://doi.org/10.1080/ 16070658.2005.11734033.

14. Serwadda D, Mugerwa RD, Sewankambo NK, Lwegaba A, Carswell JW, Kirya GB, et al. Slim disease: a new disease in Uganda and its association with HTLV-III infection. Lancet. 1985. https://doi.org/10.1016/S01406736(85)90122-9.

15. Charan J, Biswas T. How to calculate sample size for different study designs in medical research? Indian J Psychol Med. 2013. https://doi.org/10.4103/ 0253-7176.116232.

16. WHO. Obesity and Overweight. http://www.who.int/mediacentre/factsheets/ fs311/en/. Published 2018. Accessed 7 Oct. 2019.

17. International Diabetes Federation. The IDF consensus worldwide definition of the metabolic syndrome. https://www.pitt.edu/ super1/Metabolic/IDF1. pdf. Published 2006. Accessed 7 Oct. 2019.

18. WHO. Q\&As on hypertension. WHO. http://www.who.int/features/qa/82/en/. Accessed 7 Oct. 2019.
19. WHO. Interim WHO Clinical Staging of HIV/AIDS and HIV/AIDS case definitions for surveillance African region. 2005. https://www.who.int/hiv/ pub/guidelines/clinicalstaging.pdf. Accessed 10 Feb. 2020.

20. Craig CL, Marshall AL, Sjöström M, Bauman AE, Booth ML, Ainsworth BE, et al. International physical activity questionnaire: 12-country reliability and validity. Med Sci Sports Exerc. 2003. https://doi.org/10.1249/01.MSS.0000078924.61453.FB.

21. Bohlmann IM, Mackinnon S, Kruger H, Leach L, van Heerden J, Cook I, et al. Is the International Physical Activity Questionnaire (IPAQ) valid and reliable in the South African population? Med Sci Sports Exercise. 2001. https://doi. org/10.1097/00005768-200105001-00672.

22. Lynch E, Liu K, Wei GS, Spring B, Kiefe C, Greenland P. The relation between body size perception and change in body mass index over 13 years: the Coronary Artery Risk Development in Young Adults (CARDIA) study. Am J Epidemiol. 2009. https://doi.org/10.1093/aje/kwn412.

23. Gelpi M, Afzal S, Lundgren J, Ronit A, Roen A, Mocroft A, et al. Higher risk of abdominal obesity, elevated low-density lipoprotein cholesterol, and hypertriglyceridemia, but not of hypertension, in people living with human immunodeficiency virus (HIV): results from the Copenhagen comorbidity in HIV infection study. Clin Infect Dis. 2018. https://doi.org/10.1093/cid/ciy146.

24. Castro Ade C, Silveira EA, Falco Mde O, Nery MW, Turchi MD. Overweight and abdominal obesity in adults living with HIV/AIDS. Rev Assoc Med Bras. 2016. https://doi.org/10.1590/1806-9282.62.04.353.

25. Ekoru K, Murphy GAV, Young EH, Delisle H, Jerome CS, Assah F, et al. Deriving an optimal threshold of waist circumference for detecting cardiometabolic risk in sub-Saharan Africa. Int J Obes (Lond). 2017. https:// doi.org/10.1038/ijo.2017.240

26. Crowther NJ, Norris SA. The current waist circumference cut point used for the diagnosis of metabolic syndrome in sub-Saharan African women is not appropriate. PLoS One. 2012. https://doi.org/10.1371/journal.pone.0048883.

27. Owolabi EO, Ter Goon D, Adenivi OV, Ajayi Al. Optimal waist circumference cutoff points for predicting metabolic syndrome among low-income black South African adults. BMC Res Notes. 2018. https://doi.org/10.1186/s13104-018-3136-9.

28. Beraldo RA, Meliscki GC, Silva BR, Navarro AM, Bollela VR, Schmidt A, et al. Anthropometric measures of central adiposity are highly concordant with predictors of cardiovascular disease risk in HIV patients. Am J Clin Nutr. 2018. https://doi.org/10.1093/ajcn/nqy049.

29. Nguyen KA, Peer N, de Villiers A, Mukasa B, Matsha TE, Mills EJ, et al. Optimal waist circumference threshold for diagnosing metabolic syndrome in African people living with HIV infection. PLOS One. 2017. https://doi.org/ 10.1371/journal.pone.0183029.

30. O'Neill T, Guaraldi G, Orlando G, Carli F, Garlassi E, Zona S, et al. Combined use of waist and hip circumference to identify abdominally obese HIVinfected patients at increased health risk. PLoS One. 2013. https://doi.org/10. 1371/journal.pone.0062538.

31. Semu H, Zack RM, Liu E, Hertzmark E, Spiegelman D, Sztam K, et al. Prevalence and risk factors for overweight and obesity among HIV-infected adults in Dar es Salaam, Tanzania. J Int Assoc Provid AIDS Care. 2016. https://doi.org/10.1177/2325957414542574.

32. WHO. Reducing consumption of sugar-sweetened beverages to reduce the risk of unhealthy weight gain in adults. https://www.who.int/elena/titles/ bbc/ssbs_adult_weight/en/. Accessed 1 Feb 2020.

33. WHO. Global status report on noncommunicable diseases 2014. https:/apps. who.int/iris/bitstream/handle/10665/148114/9789241564854_eng.pdf;jsessionid $=$ 4B5FEC3B4BB3E8E6B740F6023A989E3A? sequence $=1$. Accessed 7 Oct 2019.

34. Mariz Cde A, Albuquerque Mde F, Ximenes RA, Melo HR, Bandeira F, Oliveira $\mathrm{TG}$, et al. Body mass index in individuals with HIV infection and factors associated with thinness and overweight/obesity. Cad Saude Publica. 2011. https://doi.org/10.1590/s0102-311×2011001000013.

35. Crum-Cianflone NF, Roediger M, Eberly LE, Ganesan A, Weintrob A, Johnson E, et al. Impact of weight on immune cell counts among HIV-infected persons. Clin Vaccine Immunol. 2011. https://doi.org/10.1128/CVI.00020-11.

36. Huttunen R, Syrjänen J. Obesity and the risk and outcome of infection. Int J Obes (Lond). 2013. https://doi.org/10.1038/ijo.2012.62.

\section{Publisher's Note}

Springer Nature remains neutral with regard to jurisdictional claims in published maps and institutional affiliations. 